\title{
HE4 protein and SMRP: Potential novel biomarkers in ovarian cancer detection
}

\author{
ANNA FRITZ-RDZANEK ${ }^{1}$, WOJCIECH GRZYBOWSKI ${ }^{1}$, JAROSŁAW BETA ${ }^{1}$, \\ ANDRZEJ DURCZYŃSKI ${ }^{1}$ and ARTUR JAKIMIUK ${ }^{1-3}$ \\ ${ }^{1}$ Department of Obstetrics and Gynecology, Central Clinical Hospital of Ministry of Interior and Administration, \\ 02-507 Warsaw; ${ }^{2}$ Centre for Reproductive Medicine, Institute of Mother and Child, 01-001 Warsaw; \\ ${ }^{3}$ Department of Surgical Research and Transplantology, Medical Research Center, \\ Polish Academy of Sciences, 02-105 Warsaw, Poland
}

Received January 27, 2012; Accepted May 16, 2012

DOI: $10.3892 / \mathrm{ol} .2012 .757$

\begin{abstract}
Epithelial ovarian cancer has the highest mortality of all gynecological cancers, and its progression is often without symptoms. Clinical outcome and survival may be improved if the disease is identified in the early stages. The objective of the study was to evaluate the utility of the serum biomarkers human epididymis protein 4 (HE4), soluble mesothelin-related protein (SMRP) and CA125 in the detection of ovarian cancer. In this retrospective study, the serum concentrations of CA125, HE4 protein and SMRP were measured in a cohort of 70 patients with epithelial ovarian cancer (EOC) compared with 78 healthy controls. Median serum levels of CA125 for ovarian cancer cases were $503.55 \pm 560.7 \mathrm{U} / \mathrm{ml}$ vs. $9.28 \pm 14.47 \mathrm{U} / \mathrm{ml}$ in the control group $(\mathrm{p}<0.001)$; for SMRP 5.13 $\pm 7.64 \mathrm{nM}$ vs. $1.02 \pm 0.89 \mathrm{nM}(\mathrm{p}<0.01)$; and for HE4 597.95 \pm 934.59 pM vs. $56.75 \pm 43.79$ pM $(\mathrm{p}<0.001)$, respectively. Positive correlations between the clinical stage of EOC and CA125, HE4 and SMRP serum concentrations were found $[(\mathrm{R}=0.83 ; \mathrm{p}<0.001) ;(\mathrm{R}=0.64 ; \mathrm{p}<0.001) ;(\mathrm{R}=0.45$; $\mathrm{p}<0.001)$, respectively]. Data analysis for the whole study group also revealed a significant correlation between plasma concentrations of CA125 and HE4 ( $\mathrm{R}=0.45$; $\mathrm{p}<0.001)$, between CA125 and SMRP $(\mathrm{R}=0.38 ; \mathrm{p}<0.001)$ as well as HE4 and SMRP $(\mathrm{R}=0.51 ; \mathrm{p}<0.001)$. Similar significant correlations between serum biomarker concentrations were also found in the ovarian cancer group [CA125 and HE4 $(\mathrm{R}=0.31 ; \mathrm{p}<0.01)$; CA125 and SMRP $(\mathrm{R}=0.25 ; \mathrm{p}<0.05)$; HE4 and SMRP $(\mathrm{R}=0.44, \mathrm{p}<0.001)$, respectively]. A significant correlation was observed between the serous histological type of EOC and serum concentration of HE4 in the study group compared with other non-serous types of ovarian cancer $(\mathrm{p}<0.01)$. In conclu-
\end{abstract}

Correspondence to: Professor Artur Jakimiuk, Woloska Street 137, 02-507 Warsaw, Poland

E-mail: jakimiuk@yahoo.com

Key words: ovarian cancer, biomarkers, human epididymis protein 4 , soluble mesothelin related protein, CA125 sion, measuring CA125 in combination with new biomarkers such as SMRP and HE4 may improve the accuracy of ovarian cancer diagnosis, particularly in early detection of the disease.

\section{Introduction}

Epithelial ovarian cancer (EOC) has the highest mortality of all gynecological cancers and is the fifth leading cause of mortality due to cancer among women. Despite relatively low incidence (approximately 1/100 000 new cases per year), EOC presents a high case-to-fatality ratio. That progression of the disease is relatively symptomless makes a significant contribution to the high mortality rates. The 5-year survival rate for EOC is approximately $45 \%$, largely due to the high proportion of cancer cases that are not detected until they have spread beyond the ovary to the pelvis and upper abdomen. Early stages of the disease remain without symptoms, with the first signs typically occurring at an advanced stage. Thus, ovarian cancer is commonly known as a 'silent killer'. Clinical outcome and survival may be significantly improved by identifying the disease in its early stages without the need for altering surgical or chemotherapeutic approaches. Although certain ovarian cancer screening tests have been shown to decrease mortality rates, the possibility of efficient screening that may be used in everyday practice remains elusive. Measurement of serum CA125 antigen remains the gold standard (1-18).

The best-studied serum marker for ovarian cancer, CA125, is elevated in less then half of early stage EOC cases and is not expressed in approximately $20 \%$ of ovarian cancers resulting in a decrease of sensitivity. The lack of specificity of CA125 is secondary to its levels being elevated in a number of benign gynecological and non-gynecological conditions. As such, in recent years over 45 new or already known substances have been verified as ovarian cancer biomarkers, such as human epididymis protein 4 (HE4) and soluble mesothelin-related protein (SMRP). Novel biomarkers alone or combined with CA125 potentially increase the sensitivity and specificity of CA125. Mesothelin and HE4 protein are the most intensively studied of the novel biomarkers (19-28). 
Table I. Demographics and clinical characteristics of study population.

\begin{tabular}{lcc}
\hline Characteristics & Study group $(\mathrm{n}=70)$ & Control group (n=78) \\
\hline Age (years) & & $55.06(11.21)$ \\
Mean (SD) & $57.36(9.73)$ & $32-74$ \\
Range & $20-80$ & $27(34.6 \%)$ \\
Menopausal status & & $51(65.4 \%)$ \\
Premenopausal & $15(21.4 \%)$ & \\
Postmenopausal & $55(78.6 \%)$ & NA \\
Ovarian cancer stage (FIGO) & & NA \\
I & $15(21.4 \%)$ & NA \\
II & $3(4.3 \%)$ & NA \\
III & $45(64.3 \%)$ & NA \\
IV & $7(10 \%)$ & NA \\
Histology & & NA \\
Serous & $52(74.3 \%)$ & NA \\
Endometrioid & $5(7.1 \%)$ & $7(10 \%)$ \\
Mucinous & $6(8.6 \%)$ & \\
Other subtype & & \\
\hline
\end{tabular}

SD, standard deviation; NA, not applicable.

Mesothelin is a differentiation antigen highly expressed in mesotheliomas, pancreatic, ovarian and other types of cancer. Its expression in normal human tissues is limited to mesothelial cells lining the pleura, pericardium and peritoneum. Since the widely accepted model of ovarian carcinogenesis is the tumor origin from the ovarian surface epithelium (OSE) followed by spread to the pelvic and abdominal cavities afterwards, it appears that proteins, such as mesothelin may be useful in the early detection of ovarian cancer. Mesothelin and its soluble isoforms (SMRP) are synthesized by mesothelial cells but only in a state of serious pathology such as carcinogenesis. Ovarian cancer is one of the tumors triggering SMRP synthesis, thus a diagnostic test based on serum SMRP concentrations may be helpful in the diagnostic process $(4,24,26,29-37)$.

HE4 protein was first identified in the epithelium of the distal epididymis and was predicted to be involved in sperm maturation as a protease inhibitor. This protein has a WAP-type four-disulphide core (WFDC) domain and is encoded by the WFDC2 gene. In malignant ovarian neoplasms upregulation of that gene was observed. In patients with ovarian tumors, sensitivity of the HE4 protein is similar to CA125 but specificity for malignancy was increased as compared to benign disease $(4,24,26,38-44)$.

HE4 is not elevated in common benign gynecological and other medical conditions, whereas CA125 is elevated. SMRP and HE4 complement CA125 in ovarian cancer detection and are therefore of particular interest for the detection of early stage EOC $(26,43,44)$.

The aim of the present study was to evaluate the usefulness of the potential biomarkers SMRP and HE4 for ovarian cancer diagnosis. Additionally, the associations between SMRP, HE4 and CA125 were analyzed to evaluate the role of the two biomarkers in the early stages of the disease as well as possibilities of supporting the diagnostics of advanced stages of ovarian cancer. A further aim of the study was to investigate how the serum concentration of HE4, SMRP and CA125 varied with age, histological type and stage of ovarian cancer in women with ovarian cancer and healthy controls.

\section{Materials and methods}

Patient characteristics. Study participants were recruited between 2005 and 2007. The study group consisted of 70 patients with diagnosed ovarian cancer. The control group consisted of 78 healthy women without any pathological features of the ovaries. Prior to collection of biological samples all patients were required to provide full informed consent. Cases were defined as having epithelial carcinoma confirmed by a standardized review of the medical records and pathologist examination of paraffin-embedded tissue for tumor histology. Patients with an EOC underwent surgical staging required by protocol or tumor debulking as clinically indicated. Of the 70 patients with EOC, 52 cases had serous ovarian carcinoma, 7 cases had mucinous histological type, 5 had endometrioid EOC and 6 patients had other histological types of disease. Patient demographics and characteristics are shown in Table I. The study was approved by the local ethics committee of the Central Clinical Hospital of Ministry of Interior and Administration.

Methods. Blood $(20 \mathrm{ml})$ was collected, clotted for $60 \mathrm{~min}$ and centrifuged. The serum fraction was removed and stored at $-70^{\circ} \mathrm{C}$ until use. Serum levels of CA125, HE4 and SMRP were measured using bead-based immunoassay kits from commercial suppliers according to manufacturers' instructions.

Statistical analysis. SPSS statistical software (version 11.5; SPSS Inc., Chicago, IL, USA) was used for statistical analyses. 
The statistical tests were two-sided and considered to be statistically significant at $\mathrm{p}<0.05$.

\section{Results}

Mean values for the plasma levels of all biomarkers differed significantly between patients with cancer compared to the control group. The median serum level of CA125 for ovarian cancer cases was $503.55 \pm 560.7 \mathrm{U} / \mathrm{ml}$ vs. $9.28 \pm 14.47 \mathrm{U} / \mathrm{ml}$ in the control group $(\mathrm{p}<0.001)$. For SMRP, the median serum concentration in the study group was $5.13 \pm 7.64 \mathrm{nM}$ vs. $1.02 \pm 0.89 \mathrm{nM}$ in the control group $(\mathrm{p}<0.01)$; and for HE4 these results were $597.95 \pm 934.59 \mathrm{pM}$ in the ovarian cancer group vs. $56.75 \pm 43.79 \mathrm{pM}$ in the control group $(\mathrm{p}<0.001)$.

Data analysis also revealed significantly higher concentrations of CA125, HE4 and SMRP in the late stage ovarian cancer group (FIGO II-IV) in comparison with the early stage patients (FIGO I stage) and control group. A positive correlation between the clinical stage of ovarian cancer and CA125 blood serum concentration was found $(\mathrm{R}=0.83$; $\mathrm{p}<0.001)$. A positive correlation was found between the HE4 protein serum concentration and EOC stage $(\mathrm{R}=0.64$; $\mathrm{p}<0.001$ ) (Fig. 1) and between the SMRP plasma concentration and stage of ovarian cancer patients $(\mathrm{R}=0.45 ; \mathrm{p}<0.001)$ (Fig. 2).

Data analysis from the study participants also demonstrated a significant correlation between plasma concentration of CA125 and HE4 ( R=0.45; p<0.001), between CA125 and SMRP $(R=0.38 ; \mathrm{p}<0.001)$ as well as HE4 and SMRP $(\mathrm{R}=0.51$; $\mathrm{p}<0.001)$. Moreover, in the group of patients with ovarian cancer, similar significant correlations between serum biomarker concentrations were observed [CA125 and HE4 $(\mathrm{R}=0.31 ; \mathrm{p}<0.01)$; CA125 and SMRP $(\mathrm{R}=0.25 ; \mathrm{p}<0.05)$; HE4 and $\operatorname{SMRP}(\mathrm{R}=0.44, \mathrm{p}<0.001)]$.

Significant correlations were observed between the serous histological type of EOC and serum concentration of HE4 observed in the study group in comparison with other non-serous types of ovarian cancer $(\mathrm{p}<0.01)$ (Fig. 3).

To compare the results, SMRP plasma concentrations in the study group in serous and non-serous histological type of EOC were also analyzed, but the results turned out not to be statistically significant $(\mathrm{p}=0.29)$. Additionally, data analysis of the study group participants also revealed a positive correlation between the age of the patients and HE4 plasma concentration $(\mathrm{R}=0.32 ; \mathrm{p}<0.01)$, whereas the results for CA125 and SMRP were not statistically significant $(\mathrm{p}=\mathrm{NS})$.

\section{Discussion}

The novel ovarian cancer biomarkers HE4 protein and mesothelin have utility for the early detection of ovarian cancer, but the gold standard for ovarian cancer screening for evaluating CA125 measurements with a value above $35 \mathrm{U} / \mathrm{ml}$ is considered to be abnormal. The above-mentioned marker is characterized by a specificity of approximately $80 \%$ and a low false positive rate of less than $1 \%$. Serum CA125 is increased in approximately $50 \%$ of women with the stage I ovarian cancer according to FIGO, which appears unsatisfactory. However, $80 \%$ of women with ovarian cancer staged from II to IV according to FIGO increased the values of CA125.

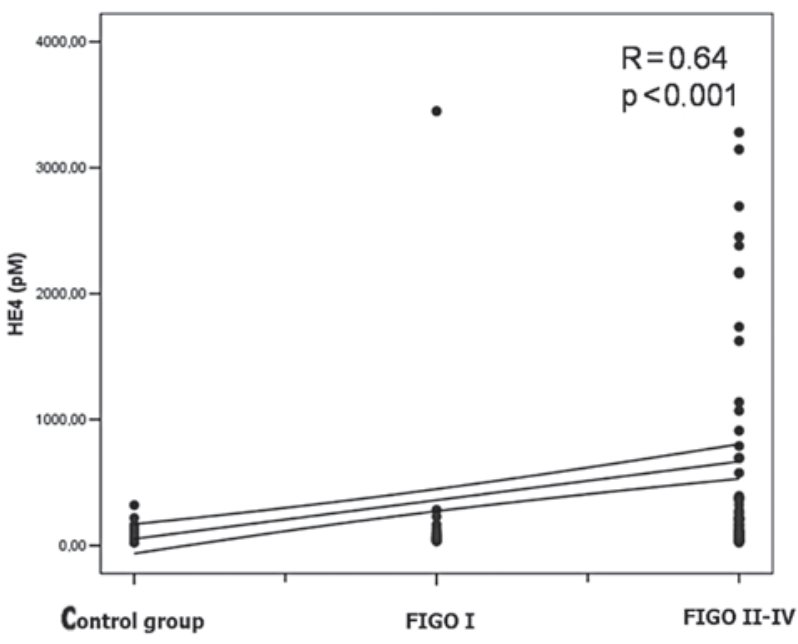

Figure 1. Positive correlation between the clinical stage of ovarian cancer and HE4 blood serum concentration in the late stage ovarian cancer group (FIGO II-IV) in comparison with early stage patients (FIGO I stage) and control group $(\mathrm{R}=0.64 ; \mathrm{p}<0.001)$.

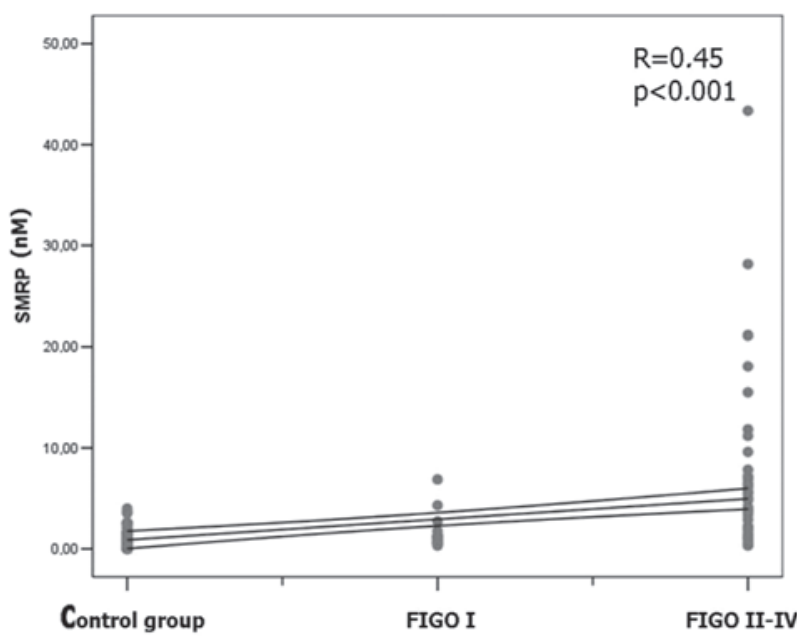

Figure 2. Positive correlation between the clinical stage of ovarian cancer and SMRP blood serum concentration in the late stage ovarian cancer group (FIGO II-IV) in comparison with early stage patients (FIGO I stage) and control group $(\mathrm{R}=0.45 ; \mathrm{p}<0.001)$.

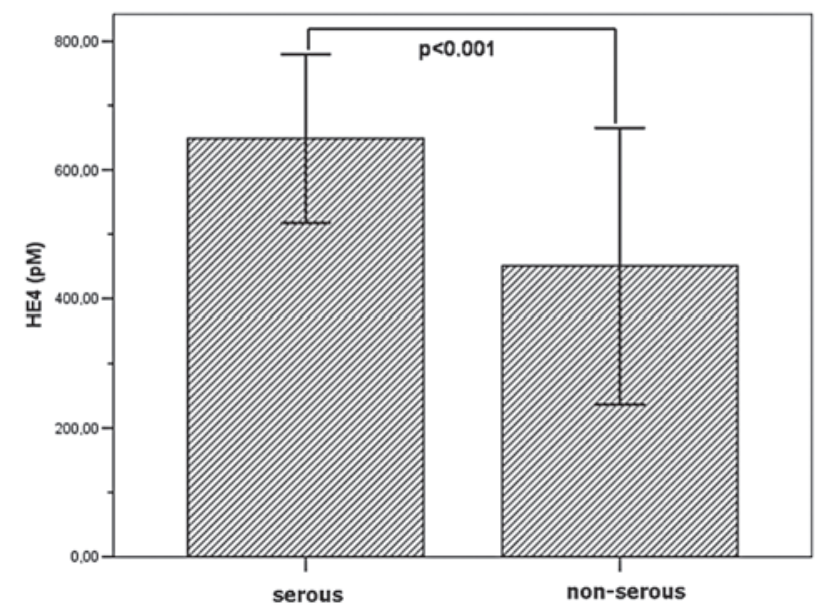

Figure 3. Mean HE4 plasma concentration in serous and non-serous histological types of ovarian cancer $(\mathrm{p}<0.01)$ 
Nonetheless, screening based only on CA125 measurements, results in approximately $20 \%$ of cases of ovarian cancer not being detected $(36,37)$.

Furthermore, in a study by Pauler et al, the authors reported that CA125 levels in healthy women vary based on characteristic such as age, race, smoking status, caffeine intake, age at menarche and menopause status $(26,45)$.

SMRP and HE4 are two of the most promising novel ovarian cancer biomarkers and are candidates for early detection markers to replay or complement CA125 (4).

In a study by McIntosh et al, specificity of CA125 alone was found to be $79 \%$, increasing to $87 \%$ when CA125 is used with the composite marker mesothelin (sensitivity at 98\%). However, the authors concluded that, as a single marker, CA125 is a better alternative to SMRP (36).

The present study found a significant correlation between the clinical stage of the ovarian cancer and CA125, HE4 and SMRP blood serum concentration in the late stage ovarian cancer group (FIGO II-IV) in comparison with early stage patients (FIGO I stage) and a control group. This is in accordance with data presented in the literature $(28,43,44,46,47)$.

The results suggest that HE4 and SMRP improved performance in early stage disease, but since the majority of the cases (in our study 74.3\%) were diagnosed in late stages of the disease (III or IV FIGO), larger study cohorts are required to confirm this hypothesis. Study results have shown a significant correlation between the serous histological type of EOC and serum concentration of HE4 in the study group in comparison with other non-serous types of ovarian cancer $(p<0.01)$. However, for SMRP and CA125, the results turned out not to be statistically significant $(p=0.29 ; p=0.09$ ). Findings of a study by Palmer et al showed results for the HE4 protein to be statistically significant. Those authors concluded that sensitivity and specificity of single marker HE4 is similar to the combination of CA125 and HE4 and higher than that of CA125 alone (28).

In a study by Shah et al, mesothelin and HE4 plasma levels were found to be higher for mucinous ovarian cancer cases. However, those results may have been spurious due to the small total number of mucinous cancer cases in the cohort $(n=6)(26)$. In our study, no such correlation for the mucinous histological type of cancer was found, but there were only seven cases of this type in our cohort. Moreover, in the study by Palmer $e$ t al extremely low results were obtained for mucinous type sensitivity and specificity for the combination of CA125 and HE4 (17 and 98\%, respectively) $(26,28)$.

We also observed a correlation between the plasma concentration of HE4 and age in the cancer subgroup. Similar results were published in 2008 by Lowe et al whereby levels of HE4 serum increased with age in a similar manner between women with ovarian cancer and healthy controls. For SMRP and CA125, the results were found not to be statistically significant (4).

However, most recent studies undermine the role of CA125 as a single biomarker in early detection of ovarian cancer. Investigators have attempted to increase the sensitivity of the test without decreasing its specificity. It appears that the combination of CA125 with other blood serum biomarkers such as SMRP or HE4 may be useful $(31,32,48,49)$.

The main conclusion derived from the present study and supported by previous studies is that HE4 protein, especially in combination with CA125 and SMRP, may be used as a marker of early stage ovarian cancer. Serum SMRP and HE4 may be useful biomarkers for ovarian cancer screening and detection. Although it cannot be used alone in early stages of the disease, SMRP and HE4 assessment is a useful screening tool in advanced stages of ovarian cancer. Serum CA125 antigen is currently the most efficient ovarian cancer biomarker, especially in early stages of the disease. Data from the present study suggest that HE4 and SMRP is a potentially useful in multiple marker screening for the serous type of ovarian carcinoma. Analysis of the biomarkers also demonstrated age-dependent differences in the serum concentrations of HE4, as previously reported $(4,44)$. Further studies are required to assess this property of HE4 and SMRP alone and confirm their performance at high specificity before we are able to make statements concerning the superiority or inferiority of these new biomarkers over CA125 alone for early detection.

Ongoing efforts using targeted discovery, combination of markers, and stratification of screening populations by cancer risk may yet lead to an effective early detection test for ovarian cancer. However, ovarian cancer is a heterogeneous disease and it may be difficult for a single marker, even with serial testing, to achieve a level of robust performance that would allow cost-effective screening of a general population. It is conceivable that by incorporating existing or newly discovered tumor markers one could significantly improve the sensitivity and specificity of detection of true early signs of ovarian cancer.

\section{Acknowledgements}

This study was supported by the Institute of Mother and Child in Warsaw, Poland by grant number 510-31-82.

\section{References}

1. Jemal A, Murray T, Ward E, et al: Cancer statistics. CA Cancer J Clin 55: 10-30, 2005.

2. Tortorelo-Luna G and Mitchell M: The epidemiology of ovarian cancer. J Cell Bioch 23: 200-207, 1995.

3. Gupta D and Lis C: Role of CA125 in predicting ovarian cancer survival - a review of the epidemiological literature. J Ovarian Res 2: 13, 2003.

4. Lowe K, Shah C, Wallace E, et al: Effects of personal characteristics on serum CA125, mesothelin and HE4 levels in healthy postmenopausal women at high-risk for ovarian cancer. Cancer Epidemiol Biomarkers Prev 17: 2480-2487, 2008.

5. Chu C and Rubin S: Screening for ovarian cancer in the general population. Best Pract Res Clin Obstet Gynaecol 20: 307-320, 2002.

6. Canevari S, Gariboldi M, Reid J, et al: Molecular predictors of response and outcome in ovarian cancer. Crit Rev Oncol Hematol.60: 19-37, 2006.

7. Gilks C, Ionescu D, Kalloger S, et al: Tumor cell type can be reproducibly diagnosed and is of independent prognostic significance in patients with maximally debulked ovarian carcinoma. Hum Pathol 39 (8): 1239-1251, 2008.

8. Pettersson F: Annual report on the results of treatment in gynecological cancer. Stockholm, International Federation of Gynecology and Obstetrics, 1994.

9. Lu K, Patterson A, Wang L, et al: Selection of potential markers of epithelial ovarian cancer with gene expression arrays and recursive descent partition analysis. Clin Cancer Res 10: 3291-3300, 2004.

10. Hoskins W: Prospective on ovarian cancer: why prevent? Cell Biochem Suppl 23: 189-199, 1995.

11. Pettersson F, Kolstad P, Ludwig H, et al: Annual report on the results of treatment in gynecological cancer. International Federation of Gynecology and Obstetrics, 1998. 
12. Nguyen $H$, Averette $H$, Hoskins $W$, et al: National survey of ovarian carcinoma VI. Critical assessment of current International Federation of Gynecology and Obstetrics staging system. Cancer 72: 3007-3011, 1993

13. Bast RC Jr, Brewer M, Zou C, et al: Ovarian cancer prevention and early detection: mission impossible? Recent Results Cancer Res 174: 91-900, 2007.

14. Zhang Z, Yu Y, Xu F, et al: Combining multiple serum tumor markers improves detection of stage I epithelial ovarian cancer. Gynecol Oncol 107: 526-531, 2007.

15. Havrilesky L, Whitehead C, Rubatt J, et al: Evaluation of biomarker panels for early stage ovarian cancer detection and monitoring for disease recurrence. Gynecol Oncol 110: 374-382, 2008.

16. Scholler N, Crawford M, Sato A, et al: Bead-based ELISA assays for validation of ovarian cancer early detection markers. Clin Cancer Res 1: 2117-2124, 2006.

17. Nosov V, Feng S, Malaika A, et al: Validation of serum biomarkers for detection of early-stage ovarian cancer. Am J Obstet Gynecol 200: 639 e1-5, 2009.

18. Oikonomopolou K, Li L, Zheng Y, et al: Prediction of ovarian cancer prognosis and response to chemotherapy by a serum-based multiparametric biomarker panel. Br J Cancer 99: 1103-1113, 2008.

19. Pauler D, Menon U, McIntosh M, et al: Factors influencing serum Ca125II levels in healthy postmenopausal women. Cance Epidemiol Biomarkers Prev 10: 489-493, 2001.

20. Bast R and Knapp R: Use of CA125 antigen in diagnosis and monitoring of ovarian carcinoma. Eur Obset Gyncol Reprod Biol 19: 354-356, 1995

21. Fishman D: The present and future of biomarkers for the early detection of epithelial ovarian carcinoma. CME J Gyneco Oncol 4: 33-36, 1999.

22. Jacobs I and Bast R: The Ca125 tumor-associated antigen: a review of literature. Human Reprod 4: 1-12, 1989.

23. Wollas R, Xu F, Jacobs I, et al: Elevation of multiple serum markers in patients with stage I ovarian cancer. JNCI 85 1748-1751, 1993

24. Bast R: Status of tumor markers in ovarian cancer screening. J Clin Oncol 21: 200-205, 2003.

25. Skates S, Xu F, Yu H, et al: Toward an optimal algorithm for ovarian cancer screening with longitudinal tumor markers. Cancer 76: 2004-2010, 1995.

26. Shah $\mathrm{C}$, Lowe $\mathrm{K}$, Paley $\mathrm{P}$, et al: Influence of ovarian cancer risk status on the diagnostic performance of the serum biomarkers mesothelin, HE4 and CA125. Cancer Epidemiol Biomarkers Prev 18: 1365-1372, 2009.

27. Jacobs I and Menon U: Progress and challenges in screening for early detection of ovarian cancer. Mol Cell Proteomics 3 : 355-366, 2004

28. Palmer C, Duan X, Hawley S, et al: Systematic evaluation of candidate blood markers for detecting ovarian cancer. PLoS One 3: e2633, 2008

29. Chang K and Pastan I: Molecular cloning of mesothelin, a differentiation antigen present on mesothelium, mesotheliomas, and ovarian cancers. Proc Natl Acad Sci 93 USA: 136-140, 1996.

30. Chang K, Pai LH, Batra JK, et al: Characterization of the antigen (CAK1) recognized by monoclonal antibody K1 present on ovarian cancers and normal mesothelium. Cancer Res 52: 181-186, 1992.
31. Blaustein A: Peritoneal mesothelium and ovarian surface cells-shared characteristics. Int J Gynecol Pathol 3: 361-375, 1984

32. Okamura H, Katabuchi H, Nitta M, et al: Structural changes and cell properties of human ovarian surface epithelium in ovarian pathophysiology. Microsc Res Tech 69: 469-481, 2006.

33. Dubeau L: The cell of origin of ovarian epithelial tumours Lancet Oncol 9: 1191-1197, 2008.

34. Okamura H: Detailed morphology of human ovarian surface epithelium focusing on its metaplastic and neoplastic capability. Ital J Anat Embryol 106 (Suppl 2): 263-276, 2001.

35. Kurman RJ and Shih IM: Pathogenesis of ovarian cancer: lessons from morphology and molecular biology and their clinical implications. Int J Gynecol Pathol 27: 151-160, 2008.

36. McIntosh M, Drescher C, Karlan B, et al: Combining CA125 and SMRP serum markers for diagnosis and early detection of ovarian carcinoma. Gynecol Oncol 95: 9-15, 2004.

37. Scholler N, Garvik B, Hayden-Ledbetter M, Kline T and Urban N: Development of a CA125-mesothelin cell adhesion assay as a screening tool for biologics discovery. Cancer Lett 247: 130-136, 2007.

38. Drapkin R, Von Horsten H, Lin Y, et al: Human epididymis protein 4 (HE4) is a secreted glycoprotein that is overexpressed by serous and endometrioid ovarian carcinomas. Cancer Res 65 : 2162-2169, 2005.

39. Galgano M, Hampton $\mathrm{G}$ and Frierson $\mathrm{H}$ : Comprehensive analysis of HE4 expression in normal and malignant human tissues. Mod Pathol 19: 847-853, 2006.

40. Hellström I, Raycraft J, Hayden-Ledbetter M, et al: The HE4 (WFDC2) protein is a biomarker for ovarian carcinoma. Cancer Res 63: 3695-3700, 2003.

41. Moore R, Brown A, Miller C, et al: Utility of a novel serum tumor biomarker HE4 in patients with uterine cancer. J Clin Oncol 24: 18S, 2006

42. Berry N, Cho Y, Harrington M, et al: Transcriptional targeting in ovarian cancer cells using the human epididymis protein 4 promoter. Gynecol Oncol 92: 896-904, 2004.

43. Moore R, Brown A, Miller M, et al: The use of multiple novel tumor biomarkers for the detection of ovarian carcinoma in patients with a pelvic mass. Gynecol Oncol 108: 402-408, 2007.

44. Moore R, McMeekin D, Brown A, et al: A novel multiple marker bioassay utilizing HE4 and CA125 for the prediction of ovarian cancer in patients with a pelvic mass. Gynecol Oncol 112: 40-46, 2009.

45. Pauler D, Menon U, McIntosh M, et al: Factors influencing serum CA125II levels in healthy postmenopausal woman at high-risk for ovarian cancer. Cancer Epidemiol Biomarkers Prev 17: 2480-2487, 2008.

46. Hellström I, Raycraft J, Kanan S, et al: Mesothelin variant I is released from tumor cells as a diagnostic marker. Cancer Epidemiol Biomarker Prev 15: 1014-1020, 2006.

47. Das P and Bast R Jr: Early detection of ovarian cancer. Biomark Med 2: 291-303, 2008

48. Köbel M, Kalloger S, Boyd N, et al: Ovarian carcinoma subtypes are different diseases: implication for biomarker studies. PLoS Med 5: e232, 2008.

49. Kozak K, Su F, Whitelegge J, et al: Characterization of serum biomarkers for detection of early stage ovarian cancer. Proteomics 5: 4589-4596, 2005. 